\title{
Some remarks on invariant eigendistributions on semisimple Lie groups
}

\author{
By \\ Takeshi HIRAI \\ (Received, February 15, 1972)
}

\section{Introduction}

Let $G$ be a connected real semisimple Lie group with Lie algebra g. Denote by $C_{0}^{\infty}(G)$ the set of all indefinitely differentiable functions on $G$ which vanish outside some compact sets. For a differential operator $D$ on $G$, we define its adjoint $D^{*}$ as

$$
\int_{G} D f_{1}(g) f_{2}(g) d g=\int_{G} f_{1}(g) D^{*} f_{2}(g) d g \quad\left(f_{1}, f_{2} \in C_{0}^{\infty}(G)\right)
$$

where $d g$ is a Haar measure on $G$. For any distribution $\pi$ on $G$, we put $(D \pi)(f)=\pi\left(D^{*} f\right)\left(f \in C_{0}^{\infty}(G)\right)$. A differential operator on $G$ is called Laplace operator if it is invariant under both left and right translations. As usual, let us identify every $X \in \mathrm{g}$ with a left-invariant differential operator on $G$. Then the center 3 of the universal envelopping algebra $U\left(g_{c}\right)$ of the complexification $g_{c}$ of $g$ is the algebra of all Laplace operators on $G$. The correspondence $D \rightarrow D^{*}$ on $U\left(\mathfrak{g}_{c}\right)$ is its anti-automorphism generated by $X \rightarrow-X(X \in \mathrm{g})$.

A distribution $\pi$ on $G$ is called invariant if it is invariant under any inner automorphism of $G$. It is called eigendistribution if there exists a homomorphism $\lambda$ of $\mathbf{Z}$ into $\boldsymbol{C}$ such that $Z \pi=\lambda(Z) \pi(Z \in \mathbf{3})$. Here $\lambda$ is called the infinitesimal character of $\pi$. Let $Z_{G}$ be the center of $G$. 
If there exists a homomorphism $\chi$ of $Z_{G}$ into $C^{*}$ such that $\pi(z g)=$ $\chi(z) \pi(g)\left(z \in Z_{G}\right), \pi$ is called $Z_{G}$-simple.

Now let $g \rightarrow T(g)(g \in G)$ be a representation of $G$ by bounded operators on a Hilbelt space $\mathcal{H}$. Put for any $f \in C_{0}^{\infty}(G)$,

$$
T(f)=\int_{G} T(g) f(g) d g .
$$

A representation $(T, \mathscr{H})$ is called (topologically) irreducible if $\mathscr{H}$ has no closed invariant subspace except $\{0\}$ and $\mathscr{H}$ itself. An irreducible representation $(T, \mathscr{H})$ is called quasi-simple [2(a), I] if there exist homomorphisms $\chi$ of $Z_{G}$ into $C^{*}$ and $\lambda$ of $B$ into $C$ such that

$$
T(z)=X(z) l_{\mathscr{H}}\left(z \in Z_{G}\right), \quad T(Z)=\lambda(Z) \mathfrak{l}_{\mathscr{H}^{\circ}}(Z \in \mathcal{B}),
$$

where $\mathscr{H}^{0}$ is the Gårding subspace of $\mathscr{H}$ spanned by all $T(f) v\left(f \in C_{0}^{\infty}(G)\right.$, $v \in \mathscr{H})$ and $l_{\mathscr{H}^{\circ}}$ denotes the identity operator on $\mathscr{H}^{0}$. The character $\pi$ of such representation can be defined as to be the distribution $\pi(f)$ $=\operatorname{tr}(T(f))\left(f \in C_{0}^{\infty}(G)\right)[2(\mathrm{a}), \mathrm{II}]$. Then $\pi$ is a $Z_{G^{-}}$-simple invariant eigendistribution corresponding to $\chi$ and $\lambda$. Call it simply irreducible character.

Denote by $\mathfrak{A}(\lambda)$ (or $\mathfrak{S}(\lambda)$ ) the set of all invariant eigendistributions on $G$ (or linear combinations of irreducible characters) with infinitesimal character $\lambda$. Then $\mathfrak{U}(\lambda) \supset \mathfrak{C}(\lambda)$. One of the purposes of this paper is to study the problem whether $\mathfrak{U}(\lambda)=\mathfrak{S}(\lambda)$ for all $\lambda$ or not. Here we give an elementary proof of existence on $S L(n, \boldsymbol{R})(n \geqslant 3)$ of tempered invariant eigendistributions which can not be expressed as linear combinations of irreducible characters. Moreover for $\operatorname{SL}(n, \boldsymbol{R})$, all irreducible characters and all invariant eigendistributions with certain infinitesimal characters $\lambda$ are obtained. Therefore we know exactly the difference of $\mathfrak{A}(\lambda)$ and $\mathfrak{S}(\lambda)$ for such $\lambda$. For complex classical groups $S L(n, \boldsymbol{C}), S O(2 n+1, \boldsymbol{C}), S p(n, \boldsymbol{C})$ and $S O(2 n, \boldsymbol{C})$, we see that if $n \leqslant 3, \mathfrak{A}(\lambda)=\mathfrak{S}(\lambda)$ for any $\lambda$ and that if $n \geqslant 4, \mathfrak{A}(\lambda) \neq \mathfrak{S}(\lambda)$ for some $\lambda$. 


\section{$\S 1$. Preliminary results}

Let us introduce some notations and make some general statements. Let $G, \mathfrak{g}$ be as before and $\mathfrak{h}$ a Cartan subalgebra of $\mathfrak{g}$. Denote by $P$ the set of all positive roots of $\left(\mathfrak{g}_{c}, \mathfrak{h}_{c}\right)$ with respect to a lexicographic order. A root $\alpha$ is called real (or imaginary) if it takes only real (or imaginary) values on $\mathfrak{h}$. Denote by $P_{R}$ (or $P_{I}$ ) the set of all real (or imaginary) positive roots. Let $W_{c}$ be the Weyl group of $\left(\mathfrak{g}_{c}, \mathfrak{h}_{c}\right)$. Let $H$ be the Cartan subgroup of $G$ corresponding to $\mathfrak{h}$ and $W_{G}(H)$ the factor group of the normalizer of $\mathfrak{h}$ in $G$ by the center $H_{0}$ of $H$. For any root $\alpha$, let $X_{\alpha} \in \mathrm{g}_{c}$ be its non-zero root vector and put $\operatorname{Ad}(h) X_{\alpha}$ $=\xi_{\alpha}(h) X_{\alpha}(h \in H)$. Define for $h \in H$,

$$
\text { (1. 1) } \quad \Delta^{\prime}(h)=\prod_{\alpha \in P}\left(1-\xi_{\alpha}(h)^{-1}\right), \quad \Delta_{R}^{\prime}(h)=\prod_{\alpha \in P_{R}}\left(1-\xi_{\alpha}(h)^{-1}\right) \text {. }
$$

Replacing $G$, if necessary, by a certain covering group which covers $G$ finitely many times, we may assume that there exists a connected complex semisimple Lie group $G_{c}$ with the following two properties. (a) Let $\rho$ be the half-sum of all $\alpha \in P$ and $H_{c}$ the Cartan subgroup of $G_{c}$ corresponding to $\mathfrak{h}_{c}$. Then $\xi_{\rho}(\exp X)=e^{\rho(X)}\left(X \in \mathfrak{h}_{c}\right)$ defines a one-valued function on $H_{c}$. (b) The injection $j$ of $\mathrm{g}$ into $\mathrm{g}_{c}$ can be lifted up a homomorphism $j^{\prime}$ of $G$ into $G_{c}$. The function $\xi_{\rho^{\circ}} j^{\prime}$ on $H$ is denoted again by $\xi_{\rho}$. Now put

$$
\nabla(h)=\xi_{\rho}(h) \operatorname{sign}\left(\Delta_{R}^{\prime}(h)\right) \Delta^{\prime}(h) \quad(h \in H) .
$$

Then for any $w \in W_{G}(H)$, there exists $\epsilon(w)= \pm 1$ such that $\nabla(w h)$ $=\epsilon(w) \boldsymbol{V}(h)$.

Let $G^{\prime}$ be the set of all regular elements of $G$ and put $H^{\prime}=H \cap G^{\prime}$, $G_{H}=\bigcup_{g \in G} g H^{\prime} g^{-1}$. Define for any $f \in C_{0}^{\infty}(G)$, a function $F_{f}$ on $H^{\prime}$ as

$$
F_{f}(h)=\overline{\nabla(h)} \int_{G / H_{0}} f\left(g h g^{-1}\right) d \tilde{g}
$$

where $\tilde{g}=g H_{0}, d \tilde{g}$ is an invariant measure on $G / H_{0}$ and $\bar{a}$ denotes the complex conjugate of $a \in C$. 
Let $\mathfrak{g}=\mathfrak{k}+\mathfrak{p}$ be a Cartan decomposition of $\mathfrak{g}$ and $\mathfrak{a}$ a maximal abelian subalgebra of $\mathfrak{p}$. Moreover let $\mathfrak{h}^{0}$ be a Cartan subalgebra of $\mathfrak{g}$ such that $\mathfrak{h}^{0}=\mathfrak{a}+\mathfrak{h}^{0} \cap \mathfrak{l}$. Assume that the order in the set of roots of $\left(\mathfrak{g}_{c}, \mathfrak{h}_{c}\right)$ is compatible with one in the set of roots of $\left(\mathfrak{g}_{c}, \mathfrak{a}_{c}\right)$. Put

$$
\mathfrak{n}_{c}=\sum_{\alpha \in P, \alpha \mid} C X_{\alpha}, \quad \mathfrak{n}=\mathfrak{n}_{c} \cap \mathfrak{g} .
$$

Let $K, A$ and $N$ be the analytic subgroups of $G$ corresponding to $\mathfrak{k}$, $\mathfrak{a}$ and $\mathfrak{n}$. Then $G=K A N$ is Iwasawa decomposition of $G$. The Cartan subgroup corresponding to $\mathfrak{h}^{0}$ is denoted by $H^{0}$. We see easily that for $H=H^{0}$,

$$
F_{f}(h)=\overline{\xi_{\rho}(h)} \overline{\prod_{\alpha \in P_{I}}\left(1-\xi_{\alpha}(h)^{-1}\right)} \int_{N} \int_{K / Z_{G}} f\left(k h n k^{-1}\right) d k d n
$$

where $k=k Z_{G}, d k$ and $d n$ denote appropriate invariant measures on $K / Z_{G}$ and $N$ respectively. Hence,

Lemma 1.1. For $H=H^{0}$, the function $F_{f}$ on $H^{\prime}$ can be extended to an indefinitely differentiable function on the whole $H$ with compact support.

Now let $I\left(\mathfrak{h}_{c}\right)$ be the subset of $U\left(\mathfrak{h}_{c}\right)$ consisting of all $W_{c}$-invariant elements.

Lemma 1.2. (See [2(b), p. 118] and [2(c), Th. 3].) There exists unique isomorphism $\gamma=\gamma^{\mathfrak{\eta}}$ of 3 onto $I\left(\mathfrak{h}_{c}\right)$ such that $F_{Z f}=\gamma(Z) F_{f}$ $\left(Z \in 3^{\prime}\right.$. This $\gamma$ satisfies that $\gamma\left(Z^{*}\right)=(\gamma(Z))^{*}$.

A homomorphism of $I\left(\mathfrak{h}_{c}\right)$ into $C$ is always induced by some $\mu \in \mathfrak{h}_{c}^{*}$, where $\mathfrak{h}_{c}^{*}$ is the dual of $\mathfrak{h}_{c}$. Denote this one by $\lambda_{\mu}$. Then $\lambda_{\mu}=\lambda_{\mu^{\prime}}$ if and only if $\mu^{\prime}=\sigma \mu$ for some $\sigma \in W_{c}$. We say $\lambda_{\mu}$ is regular if $\mu \neq \sigma \mu$ for all $\sigma \in W_{c}$ not equal to the identity. We sometimes identify the homomorphism $\lambda$ of 3 into $C$ and the one $\lambda \circ \gamma^{-1}$ of $I\left(h_{c}\right)$. For a fixed $\lambda$, 
let us consider an analytic function $\kappa$ on $H^{0}$ satisfying for some $\mu \in\left(\mathfrak{h}_{c}^{0}\right)^{*}$ such that $\lambda=\lambda_{\mu} \circ \gamma$ the following equations:

$$
\begin{gathered}
\kappa(w h)=\epsilon(w) \kappa(h) \quad\left(w \in W_{G}\left(H^{0}\right), h \in H^{0}\right), \\
D_{\kappa}=\lambda_{\mu}(D) \kappa \quad\left(D \in I\left(\mathfrak{h}_{c}^{0}(G)\right) .\right.
\end{gathered}
$$

Define a function $\pi$ on $G$ from $\kappa$ as follows: for any $g \notin G_{H^{0}}, \pi(g)=0$; for $g \in G_{H^{0}}, \pi(g)=\left(\boldsymbol{V}\left(h_{g}\right)\right)^{-1} \kappa\left(h_{g}\right)$, where $h_{g} \in H^{0}$ is an element such that $g=g_{0} h_{g} g_{0}{ }^{-1}$ for some $g_{0} \in G$. Consider the distribution defined as

$$
\pi(f)=<\pi, f>=\int_{G} f(g) \pi(g) d g \quad\left(f \in C_{0}^{\infty}(G)\right)
$$

Then using the above two lemmas on $F_{f}$, we obtain

Proposition 1. The distribution $\pi$ defined above is an invariant eigendistribution on $G$ with the infinitesimal character $\lambda=\lambda_{\mu} \circ \gamma$ which vanishes identically outside the closure of $G_{H^{0}}$.

Proof. Chose a Haar measure $d h$ on a Cartan subgroup $H$ appropriately, then for any integrable function $\varphi$ on $G_{H}$,

$$
\int_{G_{H}} \varphi(g) d g=\int_{H} \int_{G / H_{0}} \varphi\left(g h g^{-1}\right) d g \cdot|\nabla(h)|^{2} d h .
$$

Therefore applying this formula for $H=H^{0}$,

$$
\begin{aligned}
&\left\langle\pi, f>=\int_{H^{0}} F_{f} \cdot \kappa d h .\right. \\
&\left\langle Z \pi, f>=<\pi, Z^{*} f>\right.=\int_{H^{0}} F_{Z^{*} f^{\cdot}} \cdot d h=\int_{H^{0}} \gamma\left(Z^{*}\right) F_{f^{\cdot}} \kappa d h \\
&=\int_{H^{0}} \gamma(Z)^{*} F_{f} \cdot \kappa d h=\int_{H^{0}} F_{f} \cdot \gamma(Z) \kappa d h \\
&=\int_{H^{0}} F_{f} \cdot \lambda_{\mu}(\gamma(Z)) \kappa d h=\lambda(Z)<\pi, f>.
\end{aligned}
$$

Q.E.D. 
Denote by $\mathfrak{A}_{H^{\circ}}\left(\lambda_{\mu}\right)$ the set of all invariant eigendistributions $\pi$ obtained from the analytic functions $\kappa$ on $H^{0}$ as above. Using Lemma 2.4 in $\$ 2$, we can prove as in [3(b)] the following proposition (see [3(c)]). But this one is not used to prove that for $S L(n, R)(n \geqslant 3), \mathfrak{A}(\lambda) \neq \mathfrak{c}(\lambda)$ for some $\lambda$.

Proposition 2. The set $\mathfrak{A}_{H^{\circ}}\left(\lambda_{\mu}\right)$ is equal to the set of all invariant eigendistributions on $G$ with infinitesimal character $\lambda=\lambda_{\mu^{\circ}} \gamma$ which vanish identically outside the closure of $G_{H^{\circ}}$.

\section{$\S 2$. Review on known results}

Here we summalize some known results in the form of a certain number of lemmas. Two quasi-simple irreducible representations $T_{i}$ on $\mathscr{H}_{i}(i=1,2)$ are said to be infinitesimally equivalent [2(a), I, p.230] if the corresponding representations of $U\left(g_{c}\right)$ on $\mathcal{H}_{i}^{\infty}=\Sigma_{\delta} \mathcal{H}_{i}(\delta)$ (algebraic sum) are algebraically equivalent, where $\delta$ denotes an equivalent class of irreducible representations of $K$ and $\mathscr{H}_{i}(\delta)$ denotes the subspace consisting of all vectors transformed under $T_{i}(k)(k \in K)$ according to $\delta$. Then,

Lemma 2.1 [2(a), III]. Two quasi-simple irreducible representations of $G$ have the same character if and only if they are infinitesimally equivalent. Two unitary irreducible representations have the same character if and only if they are unitary equivalent.

Let $M$ be the centralizer of $A$ in $K$. Take $\mu^{a} \in \mathrm{a}_{c}^{*}$ and a finitedimensional irreducible representation $\nu$ of $M$. Then $L=\left(\mu^{\mathrm{a}}, \nu\right)$ defines canonically a representation of $M A N$. Inducing this one from $M A N$ to $G$, we obtain a representation $T^{L}$ on a Hilbert space $\mathcal{H}^{L}$ consisting certain vector-valued functions on $K$ (see e.g., [3(a)]). Let $\mathscr{H}_{1}$ and $\mathscr{H}_{2}$ be two closed invariant subspaces of $\mathcal{H}^{L}$ such that $\mathscr{H}_{1} \supset \mathcal{H}_{2}$. If the representation induced on $\mathcal{H}_{1} / \mathcal{H}_{2}$ is irreducible, it 
is called irreducible constituent of $T^{L}$. Then we know from Th.4 of $[2(\mathrm{a}), \mathrm{II}]$ the following

Lemma 2.2. For $G=S L(n, \boldsymbol{R})$ or a connected complex semisimple Lie group, any quasi-simple irreducible representation of $G$ is infinitesimally equivalent to an irreducible constituent of some $T^{L}$.

We use the following lemmas in $\S 5$.

Lemma 2.3. Let $T_{1}, T_{2}, \ldots, T_{d}$ be the set of quasi-simple irreducible representations of $G$ any two of which are not infinitesimally equivalent. Then their characters are linearly independent.

Lemma $2.4[2(\mathrm{~d})]$. Any invariant eigendistribution $\pi$ on $G$ coincides with a locally summable function on $G$ which is analytic on $G^{\prime}$. Moreover for every Cartan subgroup $H$, the function $\kappa^{\mathfrak{h}}=\boldsymbol{\nabla} \cdot\left(\left.\pi\right|_{H^{\prime}}\right)$ on $H^{\prime}=H \cap G^{\prime}$ can be extended to an analytic function on $H^{\prime}(R)=$ $\left\{h \in H, \Delta_{R}^{\prime}(h) \neq 0\right\}$.

Let $\lambda$ be the infinitesimal character of $\pi$ and chose $\mu^{\mathfrak{y}} \in \mathfrak{h}_{c}^{*}$ such that $\lambda=\lambda_{\mu} \mathfrak{y} \circ \gamma^{\mathfrak{n}}$. Then $\kappa^{\mathfrak{y}}$ on $H^{\prime}(R)$ satisfies the analogous equations as (1. 4) and (1. 5):

(2. 1) $\quad \kappa^{\mathfrak{\natural}}(w h)=\epsilon(w) \kappa^{\mathfrak{\natural}(h)} \quad\left(w \in W_{G}(H), h \in H^{\prime}(R)\right)$,

(2. 2) $\quad D \kappa^{\mathfrak{y}}=\lambda_{\mu} \mathfrak{h}(D) \kappa^{\mathfrak{y}} \quad\left(D \in I\left(\mathfrak{h}_{c}\right)\right)$.

Suppose that $\mathfrak{h}=\mathfrak{h}_{-}+\mathfrak{h}_{+}$, where $\mathfrak{h}_{-}=\mathfrak{h} \cap \mathfrak{t}, \mathfrak{h}_{+}=\mathfrak{h} \cap \mathfrak{p}$. Then putting $H_{-}=H \cap K, \quad H=H_{-} \exp \mathfrak{h}_{+} . \quad$ For any connected component $F$ of $H^{\prime}(R)$, take $h_{0} \in H_{\text {- }}$ on the boundary of $F$. As a solution of (2.2), $\kappa^{\mathfrak{h}}$ is expressed as

(2. 3) $\left.\quad \kappa^{\mathfrak{\eta}\left(h_{0}\right.} \exp X\right)=\sum_{\sigma \in W_{c}} p_{\sigma}(X) \exp \left\{\mu^{\mathfrak{\natural}}(\sigma X)\right\}$

if $X \in \mathfrak{h}$ is sufficiently small and $h_{0} \exp X \in F$, where $p_{\sigma}$ 's are some 
polynomial functions on $\mathfrak{h}$. If $K$ is compact or $\pi$ is $Z_{G}$-simple, all $p_{\sigma}$ can be taken as not to depend on $\mathfrak{h}_{-}$, because $F=F$ exp $\mathfrak{h}_{-}$. Define $\mathfrak{A}^{\prime}(\lambda)$ (or $\mathfrak{X}^{\prime \prime}(\lambda)$, in case when $K$ is compact) as the subset of $\mathfrak{A}(\lambda)$ consisting of such $\pi$ that for any $F$ and $H$, all $p_{\sigma}$ in the expression (2.3) can be taken as to be constants (or polynomials with constant terms zero).

\section{$\S 3$. Invariant eigendistributions on $S L(n, \boldsymbol{R})$}

In this section, let $G=S L(n, \boldsymbol{R})$ and $H^{0}$ its Cartan subgroup consisting of all diagonal matrices in $G$. Let us calculate all analytic functions $\kappa$ on $H^{0}$ satisfying (1.4) and (1.5). Denote by $d\left(a_{1}, a_{2}, \ldots, a_{n}\right)$ the diagonal matrix with diagonal elements $a_{1}, a_{2}, \ldots, a_{n}$. For $h=$ $d\left(a_{1}, a_{2}, \ldots, a_{n}\right) \in H^{0}$,

$$
\nabla(h)=\left|\prod_{i<j}\left(a_{i}-a_{j}\right)\right| .
$$

The Weyl group $W_{G}\left(H^{0}\right)$, simply denoted by $W$, is isomorphic to $W_{c}$ and to the symmetric group $\mathfrak{S}_{n}$ of order $n$ as permutation group of $a_{1}$, $a_{2}, \ldots, a_{n}$. Let $\epsilon_{j}= \pm 1(1 \leqslant j \leqslant n)$ such that $\epsilon_{1} \epsilon_{2} \ldots \epsilon_{n}=1$ and put $\epsilon=\left(\epsilon_{1}, \epsilon_{2}, \ldots, \epsilon_{n}\right)$. Denote by $H^{0}(\epsilon)$ the connected component of $H^{0}$ containing $d\left(\epsilon_{1} e^{t_{1}}, \epsilon_{2} e^{t_{2}}, \ldots, \epsilon_{n} e^{t_{n}}\right)$, where $t_{j} \in R$. Put $I_{k}=\{1,2, \ldots$, $2 k\}, J_{k}=\{2 k+1,2 k+2, \ldots, n\}$ and let $\epsilon^{(k)}$ be such row $\epsilon$ that $\epsilon_{j}=-1$ for $l \in I_{k}$ and $\epsilon_{j}=1$ for $j \in J_{k}$. Put $H_{k}^{0}=H^{0}\left(\epsilon^{(k)}\right)$. Any $H^{0}(\epsilon)$ is conjugate to some $H_{k}^{0}$ under $W$. It is sufficient to determine the restrictions $\kappa_{k}$ of $\kappa$ on $H_{k}$ for $0 \leqslant k \leqslant[n / 2]$ because for $h \in H^{0}(\epsilon)=w H_{k}^{0}$ $(w \in W), \quad \kappa(h)=\kappa_{k}\left(w^{-1} h\right)$. The subgroup $W_{k}=\left\{w \in W ; w H_{k}^{0}=H_{k}^{0}\right\}$ is isomorphic to $\mathfrak{S}_{2 k} \times \mathfrak{S}_{n-2 k}$ and (1. 4) is rewritten as

$$
\kappa_{k}(w h)=\kappa_{k}(h) \quad\left(w \in W_{k}, h \in H_{k}^{0}\right) .
$$

Any element $\mu \in\left(\mathfrak{h}_{c}^{0}\right)^{*}$ is expressed uniquely as $\mu=\left(\mu_{1}, \mu_{2}, \ldots, \mu_{n}\right)$, where $\mu_{j} \in C$ and $\mu_{1}+\mu_{2}+\ldots+\mu_{n}=0$, in such a way that

$$
\mu\left(d\left(t_{1}, t_{2}, \ldots, t_{n}\right)\right)=\sum_{1<j<n} \mu_{j} t_{j}=(\mu, t) \quad \text { (put). }
$$


To study the equations (1. 4) and (1. 5), it is convenient to replace $G$ by the reductive group $+G=\{g \in G L(n, \boldsymbol{R})$; det $g>0\}$. The results in $\S 1$ can be translated for $+G$ word for word. Denote by $+\mathfrak{h}^{0},+H^{0}$, ${ }^{+} H^{0}(\epsilon),{ }^{+} H_{k}^{0},+\nabla,{ }^{+}{ }_{\kappa}$ and ${ }^{+}{ }_{\kappa}$ the analogous objects as $\mathfrak{h}^{0}, H^{0}, H^{0}(\epsilon)$, $H_{k}^{0}, \nabla, \kappa$ and $\kappa_{k}$ respectively. Then for $h=d\left(a_{1}, a_{2}, \ldots, a_{n}\right) \in{ }^{+} H^{0}$,

$$
+\nabla(h)=\left(a_{1} a_{2} \ldots a_{n}\right)^{-\frac{n-1}{2}}\left|\prod_{i>j}\left(a_{i}-a_{j}\right)\right| .
$$

The Weyl groups are the same for $G$ and $+G$. Denote by $\boldsymbol{t}_{j}$ the differential operator $\partial / \partial t_{j}$ on $+H^{0}$. Then $I\left(+\mathfrak{h}_{c}^{0}\right)$, considered as the algebra of differential operators on $+H^{0}$, is nothing but the symmetric polynomials of $\boldsymbol{t}_{1}, \boldsymbol{t}_{2}, \ldots, \boldsymbol{t}_{n}$. For any $\mu=\left(\mu_{1}, \mu_{2}, \ldots, \mu_{n}\right) \in\left(+\mathfrak{h}_{c}^{0}\right) *$ and $D(\boldsymbol{t}) \in I\left(+\mathfrak{h}_{c}^{0}\right)$, $\lambda_{\mu}(D(t))=D(\mu)$. We restrict ourselves to treat $\mu$ such that $\mu_{1}+\mu_{2}+$ $\ldots+\mu_{n}=0$. Then for any such $\mu$, there exists a one-one correspondence between the set of all solutions $\kappa$ of the equations (1.4), (1.5) on $H^{0}$ and that of $+_{\kappa}$ of the corresponding equations on $+H^{0}$, by restricting ${ }^{+}{ }_{\kappa}$ on $H^{0}$. Therefore it is sufficient for us to study the following equations: for $0 \leqslant k \leqslant[n / 2]$,

$$
\begin{aligned}
& { }_{\kappa_{\kappa_{k}}(w h)={ }^{+} \kappa_{k}(h)}\left(h \in{ }^{+} H_{k}^{0}, w \in W_{k}\right), \\
& D(\boldsymbol{t})^{+}{ }_{\kappa_{k}}=D(\mu)^{+} \kappa_{k} \quad\left(D \in I\left({ }^{+} \mathfrak{h}_{c}^{0}\right)\right) .
\end{aligned}
$$

Put $W(\mu)=\{\tau \in W ; \tau \mu=\mu\}$ and $\bar{\sigma}=\sigma W(\mu)$ for $\sigma \in W$. Any solution of (3.6) is expressed uniquely as follows: for $h=d\left(\epsilon_{1} e^{t_{1}}, \epsilon_{2} e^{t_{2}}\right.$, $\ldots, \epsilon_{n} e^{t_{n}} \in{ }^{+} H_{k}^{0}$,

$$
+_{\kappa k}(h)=\sum_{\bar{\sigma} \in W / W(\mu)} p_{\bar{\sigma}}(t) \exp ((\sigma \mu, t)),
$$

where $p_{\bar{\sigma}}$ 's are some polynomials of $t=\left(t_{1}, t_{2}, \ldots, t_{n}\right)$. Let us rewrite (3. 5) and (3.6) in terms of $p_{\bar{\sigma}}$ 's. The equation (3. 5) is written as

$$
w p_{\bar{\sigma}}=p \overline{w \sigma} \quad\left(w \in W_{k}, \sigma \in W\right)
$$

where $w p_{\bar{\sigma}}(t)=p_{\bar{\sigma}}\left(w^{-1} t\right)$. Take a complete system $\Sigma$ of representatives 
of the double coset space $W_{k} \backslash W / W(\mu)$. Then (3.8) means that it is sufficient to determin $p_{\bar{\sigma}}$ for $\sigma \in \Sigma$ and that for any $\sigma \in \Sigma$,

$$
w p_{\bar{\sigma}}=p_{\bar{\sigma}} \quad\left(w \in W_{k} \cap W(\sigma \mu)\right)
$$

Now let $\alpha_{1}, a_{2}, \ldots, \alpha_{n}$ be the set of different numbers in $\mu_{1}, \mu_{2}, \ldots$, $\mu_{n}$ and put $A_{r}=\left\{j ; \mu_{j}=a_{r}\right\}$. Define $\sigma(i)$ as $\left(\sigma^{-1} \mu\right)_{i}=\mu_{\sigma(i)}$. Then $\sigma A_{r}=\left\{j ;(\sigma \mu)_{j}=a_{r}\right\}$. For any subset $A$ of $\{1,2, \ldots, n\}$, put

$$
D_{m}(A)=\sum_{i \in A} \boldsymbol{t}_{i}^{m}, \quad W(A)=\{w \in W ; w A=A, w(i)=i \text { for any } i \notin A\}
$$

Using the same method as in [3(b), §9], we can prove the following

Lemma 3.1. The system of equations (3.5) and (3.6) is expressed in terms of $p_{\bar{\sigma}}(\sigma \in \Sigma)$ as

(3. 10) $\left\{\begin{array}{l}w p_{\bar{\sigma}}=p_{\bar{\sigma}} \quad\left(w \in W_{k} \cap W\left(\sigma A_{r}\right), 1 \leqslant r \leqslant N\right), \\ D_{m}\left(\sigma A_{r}\right) p_{\bar{\sigma}}=0 \quad(m \geqslant 1,1 \leqslant r \leqslant N) .\end{array}\right.$

Fix $\sigma \in \Sigma$ and $r$ and put $A=\sigma A_{r} \cap I_{k}, B=\sigma A_{r} \cap J_{k}, p=p_{\bar{\sigma}}$, then $A \cap B=\phi$ and the above equations for $\sigma$ and $r$ are

$$
\left\{\begin{array}{l}
w p=p \quad(w \in W(A) \cap W(B)) \\
D_{m}(A \cap B) p=0 \quad(m \geqslant 1)
\end{array}\right.
$$

if $A$ or $B=\phi$, the polynomial $p$ does not contain the variables $t_{j}(j \in A \cap$ $B$ ) explicitely (see [3(b), §9]). If $A \neq \phi$ and $B \neq \phi$, the equation (3. 11) has the following solution:

$$
p(t)=(\$ A)^{-1} \sum_{j \in A} t_{j}-(\$ B)^{-1} \sum_{j \in B} t_{j},
$$

where $\$ A$ denotes the number of elements in $A$. Restricting this solution $p(t)$ from $+H_{k}^{0}$ to $H_{k}^{0}$, we always obtain non-zero function.

Now denote by $\mathfrak{A}_{H^{0}}^{\prime}(\lambda)$ and $\mathfrak{U}_{H^{0}}^{\prime \prime}(\lambda)$ the sets $\mathfrak{U}_{H^{0}}(\lambda) \cap \mathfrak{U}^{\prime}(\lambda)$ and 
$\mathfrak{A}_{H^{\circ}}(\lambda) \cap \mathfrak{A}^{\prime \prime}(\lambda)$. Then we obtain from the above arguments the following

Proposition 3. For $S L(n, \boldsymbol{R}), \quad \mathfrak{A}_{H_{0}}(\lambda)=\mathfrak{U}_{H^{0}}^{\prime}(\lambda)+\mathfrak{U}_{H^{0}}^{\prime \prime}(\lambda)$ (direct sum). When $n=2$, always $\mathfrak{U}_{H^{0}}^{\prime \prime}(\lambda)=\{0\}$ and $\mathfrak{U}_{H^{\circ}}(\lambda)=\mathfrak{U}_{H^{0}}^{\prime}(\lambda)$. When $n$ $\geqslant 3, \mathfrak{A}_{H^{\circ}}^{\prime \prime}(\lambda)=\{0\}$ or $\neq\{0\}$ according as $\lambda$ is regular or not.

\section{$\S 4$. Irreducible characters of $S L(n, \boldsymbol{R})$}

In this and the next sections, we calculate all irreducible characters of $G=S L(n, \boldsymbol{R})$ with certain infinitesimal characters $\lambda$. Let us apply Lem's 2.1 and 2.2. Put $\mathfrak{a}=\mathfrak{h}^{0}$ and $K=S O(n)$, then $M=$ $\left\{d\left(\epsilon_{1}, \epsilon_{2}, \ldots, \epsilon_{n}\right)\right\}$ and $M A=H^{0}$. Take $\mu=\left(\mu_{1}, \mu_{2}, \ldots, \mu_{n}\right) \in\left(\mathfrak{h}_{c}^{0}\right)^{*}$ and let $\nu=\left(\nu_{1}, \nu_{2}, \ldots, \nu_{n}\right)$ be a row of $\nu_{j}=0$ or 1 . Then $\nu$ determines a character of $M$ and the pair $(\mu, \nu)$ determines a character $\chi^{\mu, \nu}$ of $H^{0}=M A$ as

$$
\chi^{\mu, \nu}(h)=\prod_{1 \leqslant j \leqslant n}\left|a_{j}\right|^{\mu}\left(a_{j}|| a_{j} \mid\right)^{\nu},
$$

where $h=d\left(a_{1}, a_{2}, \ldots, a_{n}\right)$. Consider the induced representation of $\chi^{\mu, \nu}$ defined in $\S 2$ and denote it by $T^{\mu, \nu}$ (see also [1]). Then we see that $T^{\mu, \nu}$ and its character $\pi^{\mu, \nu}$ satisfy

$$
\begin{aligned}
& T^{\mu, \nu}(z)=\nu(z) 1 \quad\left(z \in Z_{G}\right), \quad T^{\mu, \nu}(Z)=\lambda_{\mu}(Z) 1 \quad(Z \in 3) \\
& \pi^{\mu, \nu}(z g)=\nu(z) \pi^{\mu, \nu}(g) \quad\left(z \in Z_{G}\right), \quad Z \pi^{\mu, \nu}=\lambda_{\mu}(Z) \pi^{\mu, \nu} \quad(Z \in 3) .
\end{aligned}
$$

This character is a function on $G$ which vanishes identically outside $G_{H^{0}}$ and is given on $G_{H^{0}}$ as follows:

$$
\pi^{\mu, \nu}(h)=\nabla(h)^{-1} \kappa_{\mu, \nu}^{0}(h) \quad\left(h \in H^{0} \cap G^{\prime}\right),
$$

where putting $W=W_{G}\left(H^{0}\right)$,

$$
\kappa_{\mu, \nu}^{0}(h)=\sum_{w \in W} \chi^{\mu, \nu}(w h)
$$

Therefore it follows from the results in $\S 3$ that for any $\mu \in\left(\mathfrak{h}_{c}^{0}\right)^{*}$, the 
space $\mathfrak{A}_{H^{0}}^{\prime}\left(\lambda_{\mu}\right)$ is spanned by $\pi^{\mu, \nu}$, when $\nu$ runs over all possible rows, whence $\mathbb{S}\left(\lambda_{\mu}\right) \supset \mathfrak{A}_{H^{0}}^{\prime}\left(\lambda_{\mu}\right)$. Note that $\pi^{\mu, \nu}=\pi^{\mu^{\prime}, \nu^{\prime}}$ if and only if there exists some $w \in W_{G}\left(H^{0}\right)$ such that $\chi^{\mu^{\prime}, \nu^{\prime}}(h)=\chi^{\mu, \nu}(w h) \quad\left(h \in H^{0}\right)$.

To apply Lem. 2.2, we must decompose $T^{\mu, \nu}$ into irreducible constituents. We call $\mu \in\left(\mathfrak{h}_{c}^{0}\right)^{*}$ imaginary if it takes on $\mathfrak{h}^{0}$ only pureimaginary values. If $\mu$ is imaginary, $T^{\mu, \nu}$ is unitary and its irreducibility is studied in [1]. Put $\tilde{G}=\{g \in G L(n, \boldsymbol{R})$; det $g= \pm 1\}$ and let $\tilde{H}^{0}$ be its subgroup consisting of all diagonal matrices in $\tilde{G}$. Extend $\chi^{\mu, \nu}$ from $H^{0}$ to $\tilde{H}^{0}$ by (4. 1) and construct its induced representation $\tilde{T}^{\mu, \nu}$ of $\tilde{G}$ analogously as $T^{\mu, \nu}$. Then the restriction of $\tilde{T}^{\mu, \nu}$ on $G$ is exactly $T^{\mu, \nu}$.

Lemma 4.1 [1]. The representation $\tilde{T}^{\mu, \nu}$ of $\tilde{G}$ is always irreducible if $\mu$ is imaginary.

Put $u_{0}=d(-1,-1, \ldots,-1)$ if $n$ is odd and $u_{0}=d(1,1, \ldots, 1,-1)$ if $n$ is even. Then $\tilde{G}=G \cap G u$. Using the general theory of group representations, we obtain from the above lemma the following

Lemma 4.2. When $n$ is odd, $T^{\mu, \nu}$ is always irreducible. When $n$ is even, if it is reducible, it is a direct sum of two inequivalent irreducible representations $T$ and $T^{\prime}$ such that $T^{\prime}$ is unitary equivalent to the representation $g \rightarrow T\left(u_{0} g u_{0}^{-1}\right)(g \in G)$.

Note that $\lambda_{\mu}=\lambda_{\mu^{\prime}}$ if and only if $\mu^{\prime}=\sigma \mu$ for some $\sigma \in W_{c}$. Then,

Proposition 4. Suppose $n$ is odd. If $\mu \in\left(\mathfrak{h}_{c}^{0}\right)^{*}$ is imaginary, the characters $\pi^{\mu, \nu}$ give all irreducible characters of $G$ with infinitesimal character $\lambda_{\mu}$ and $\mathfrak{E}\left(\lambda_{\mu}\right)=\mathfrak{H}_{H^{0}}^{\prime}\left(\lambda_{\mu}\right)$.

Thus, Prop's 3 and 4 give us an elementary proof of the following theorem in the case when $n$ is odd, because $\mathfrak{A}_{H^{0}}^{\prime \prime}\left(\lambda_{\mu}\right) \neq\{0\}$ for some $\lambda_{\mu}$. 
Theorem 1. For $S L(n, R)(n \geq 3)$, there exist tempered invariant eigendistributions on it which can not be expressed as linear combinations of irreducible characters (for the definition of temperedness, see $[2(\mathrm{e})])$.

Note that for $S L(n, R)$, every element in $\mathfrak{U}\left(\lambda_{\mu}\right)$ is tempered if $\mu$ is imaginary.

Apply Lem. 2.4 and consider the equations (2.1) and (2. 2) for every $H=H^{r}(0 \leqslant r \leqslant[n / 2])$. Then, using Prop. 2, we obtain

Proposition 5. When $n$ is odd, $\mathfrak{A}\left(\lambda_{\mu}\right)=\mathfrak{A}_{H^{0}}\left(\lambda_{\mu}\right)$ if $\mu \in\left(\mathfrak{h}_{c}^{0}\right)^{*}$ is imaginary.

\section{$\S 5$. Irreducible characters of $S L(n, \boldsymbol{R})$ for even $n$}

Now suppose $n=2 s$ is even. To calculate all irreducible characters, we apply Lem's 2.3 and 2.4. Put

$$
u(\theta)=\left(\begin{array}{rr}
\cos \theta & -\sin \theta \\
\sin \theta & \cos \theta
\end{array}\right)
$$

and let

$$
d\left(e^{\tau_{1}} u\left(\theta_{1}\right), e^{\tau_{2}} u\left(\theta_{2}\right), \ldots, e^{\tau_{r}} u\left(\theta_{r}\right), \epsilon_{1} e^{t_{1}}, \ldots, \epsilon_{2} e^{t_{2}}, \ldots, \epsilon_{n-2 r} e^{t_{n-2 r}}\right)
$$

be the blockwise diagonal matrix with $r$ blocks of $2 \times 2$. Denote by $H^{r}$ the set all such matrices in $G$. Then $H^{0}, H^{1}, \ldots, H^{r}$ form a complete system of Cartan subgroups of $G$ which are not conjugate to each other under inner automorphisms.

Suppose that $\mu \in\left(\mathfrak{h}_{c}^{0}\right)^{*}$ is imaginary as before and $T^{\mu, \nu}$ is reducible. Let $T, T^{\prime}$ be as in Lem. 4.2 and let $\pi, \pi^{\prime}$ be their characters. Then,

$$
\pi+\pi^{\prime}=\pi^{\mu, \nu}, \quad \pi^{\prime}(g)=\pi\left(u_{0} g u_{0}^{-1}\right) \quad\left(g \in G^{\prime}\right) .
$$

When $0 \leqslant r<s, u_{0} h u_{0}^{-1}=h$ for any $h \in H^{r}$, whence $\pi^{\prime}(h)=\pi(h)$ on $H^{r^{\prime}}=H^{r} \cap G^{\prime}$. Therefore, 


$$
\left\{\begin{array}{l}
\pi=\pi^{\prime}=2^{-1} \pi^{\mu, \nu} \text { on } H^{0^{\prime}} \\
\pi=\pi^{\prime}=2^{-1} \pi^{\mu, \nu}=0 \text { on } H^{r^{\prime}} \text { for } 0<r<s .
\end{array}\right.
$$

Moreover, since $T$ is not equivalent to $T^{\prime}, \pi \neq \pi^{\prime}$. Hence $\pi=-\pi^{\prime} \neq 0$ on $H^{s}$.

We note here that it follows from (5. 2) that any non-zero element in $\mathfrak{U}_{H^{\circ}}^{\prime \prime}\left(\lambda_{\mu}\right)$ can not be expressed as a linear combination of irreducible characters, which proves Th. 1 in the case when $n$ is even.

On the other hand, a study of the equations (2. 1), (2. 2) for $\lambda=$ $\lambda_{\mu^{\circ} \gamma}$ on $H=H^{r}(0 \leqslant r \leqslant s)$ gives us more exact results. Denote in general a solution $\kappa^{\mathfrak{h}}$ on $H=H^{r}$ by $\kappa^{r}$. For $0<r<s$, always $\kappa^{r}=0$ on $H^{r^{\prime}}(R)$. Let $M^{s}$ be the set of all $\mu^{\prime}=\left(\mu_{1}^{\prime}, \mu_{2}^{\prime}, \ldots, \mu_{n}^{\prime}\right) \in\left(\mathfrak{h}_{c}^{0}\right)^{*}$ such that $\mu_{1}^{\prime}=\mu_{2}^{\prime}, \mu_{3}^{\prime}=\mu_{4}^{\prime}, \ldots, \mu_{2 s-1}^{\prime}=\mu_{2 s}^{\prime}$. When $\sigma \mu \notin M_{s}$ for any $\sigma \in W_{c}$, always $\kappa^{s}=0$ on $H^{s^{\prime}}(R)=H^{s}$. When $\mu^{\prime}=\sigma_{0} \mu \in M_{s}$ for some $\sigma_{0} \in W_{c}$, $\boldsymbol{\kappa}^{\delta}$ is a constant multiple of

$$
\eta(h)=2^{s-1} \prod_{\sigma \in \mathbb{S}_{s}} \exp \left\{\left(\mu_{2 i-1}^{\prime}+\mu_{2 i}^{\prime}\right) \tau_{\sigma(j)}\right\}
$$

where $h=d\left(e^{\tau_{1}} u\left(\theta_{1}\right), e^{\tau_{2}} u\left(\theta_{2}\right), \ldots, e^{\tau_{s}} u\left(\theta_{s}\right)\right)$.

Let $\pi^{\mu, \nu^{i}}\left(1 \leqslant i \leqslant N_{0}\right)$ be the set of all different $\pi^{\mu, \nu}$. Then it follows from the above arguments and Lem. 2.3 that $\operatorname{dim} \subseteq\left(\lambda_{\mu}\right) \leqslant N_{0}+1$ and that at most one $T^{\mu, \nu^{i}}$ is reducible.

Suppose $\mu \in M_{s}$ and $\nu^{1}=(1,0,1,0, \ldots, 1,0)$. Let us prove that $T^{\mu, \nu^{1}}$ is reducible. Put

$$
{ }^{+} G_{2}=\{\delta \in G L(2, \boldsymbol{R}) ; \operatorname{det} \delta>0\}, D_{s}=\left\{d\left(\delta_{1}, \delta_{2}, \ldots, \delta_{s}\right) \in G ; \delta_{j} \in{ }^{+} G_{2}\right\},
$$

and $P_{s}=D_{s} N$. Denote by $D_{1 / 2, c}^{ \pm}$the irreducible unitary representations of $+G_{2}$ with the following characters respectively: for $d\left(\epsilon e^{t_{1}}, \epsilon e^{t_{2}}\right)$ and $e^{\tau} u(\theta) \in+G_{2}$,

$$
\frac{\epsilon e^{c\left(t_{1}+t_{2}\right)}}{\left|e^{\frac{t_{1}-t_{2}}{2}}-e^{-t_{1}-t_{2}}\right|^{2}} \mid \text { and } \frac{\mp e^{2 c \tau}}{e^{i \theta}-e^{-i \theta}}
$$

Consider the representation $L$ of $D_{s}$ obtained from the Kronecker 
product $\mathrm{D}_{1 / 2, \mu_{1}}^{\beta_{1}} \otimes \mathrm{D}_{1 / 2, \mu_{3}}^{\beta_{2}} \otimes \ldots \otimes \mathrm{D}_{1 / 2, \mu_{2 s-1}}^{\beta_{s}}$ with $\beta_{j}= \pm$. Extend $L$ to the parabolic subgroup $P_{s}$ and induce it from $P_{s}$ to $G$, then we obtain a unitary representation $T^{L}$ of $G($ see $[3(\mathrm{a})])$. Its character $\pi^{L}$ is given by Th. 2 in [3(a)] as follows. Let $q$ be the number of $\beta_{j}$ such that $\beta_{j}=+$. Then,

$$
\pi^{L}=\left\{\begin{array}{cc}
(-1)^{q \nabla-1} \cdot \eta & \text { on } H^{s} ; \\
0 & \text { on } H^{r}(0<r<s) \\
2^{-1} \pi^{\mu, \nu^{1}} & \text { on } H^{0} .
\end{array}\right.
$$

Let $T_{ \pm}^{\mu}$ be the induced representations for which $\beta_{1}= \pm, \beta_{2}=\beta_{3}=\ldots$ $=\beta_{s}=+$ and let $\pi_{ \pm}^{\mu}$ be their characters. Then $\pi_{+}^{\mu}+\pi_{-}^{\mu}=\pi^{\mu, \nu 1}$. Therefore $T^{\mu, \nu^{1}}$ is equivalent to the direct sum of $T_{+}^{\mu}$ and $T_{-}^{\mu}$ and the latters are irreducible. Thus,

Proposition 6. Suppose $n=2 s$ is even and $\mu \in\left(\mathfrak{h}_{c}^{0}\right)^{*}$ is imaginary. (a) When $\sigma \mu \notin M_{s}$ for any $\sigma \in W_{c}$, all $T^{\mu, \nu}$ are irreducible and $\pi^{\mu, \nu}$ 's give all irreducible characters of $G$ with infinitesimal character $\lambda_{\mu}$ and $\mathfrak{S}\left(\lambda_{\mu}\right)=\mathfrak{A}_{H^{0}}^{\prime}\left(\lambda_{\mu}\right)$. (b) When $\mu \in M_{s}$, let $\pi^{\mu, \nu i}\left(1 \leqslant i \leqslant N_{0}\right)$ be all different $\pi^{\mu, \nu}$ and $\gamma^{1}=(1,0,1,0, \ldots, 1,0)$. Then $T^{\mu, \nu^{1}}$ is equivalent to the direct sum of $T_{+}^{\mu}$ and $T_{-}^{\mu}$ and all other $T^{\mu, \nu^{i}}$ are irreducible. All irreducible characters on $G$ with infinitesimal character $\lambda_{\mu}$ are $\pi_{+}^{\mu}, \pi_{-}^{\mu}$ and $\pi^{\mu, \nu^{i}}(i \neq 1)$, and $\mathfrak{S}\left(\lambda_{\mu}\right)=\mathfrak{U}_{H^{0}}^{\prime}\left(\lambda_{\mu}\right)+C\left(\pi_{+}^{\mu}-\pi_{-}^{\mu}\right)$.

Analogously as Prop. 5, we obtain also

Proposition 7. Suppose that $\mu \in\left(\mathfrak{h}_{c}^{0}\right)^{*}$ is imaginary. In the case (a), $\mathfrak{A}\left(\lambda_{\mu}\right)=\mathfrak{A}_{H^{0}}\left(\lambda_{\mu}\right)$. In the case (b), $\mathfrak{A}\left(\lambda_{\mu}\right)=\mathfrak{A}_{H^{0}}\left(\lambda_{\mu}\right)+C\left(\pi_{+}^{\mu}-\pi_{-}^{\mu}\right)$.

Moreover we can prove for $S L(n, \boldsymbol{R})$ the following generalization of Prop. 3(cf. [3(c)]). 
Proposition 8. For any homomorphism $\lambda$ of 3 into $C, \mathfrak{A}(\lambda)=$ $\mathfrak{U}^{\prime}(\lambda)+\mathfrak{U}^{\prime \prime}(\lambda)$ (direct sum) and $\mathfrak{S}(\lambda) \supset \mathfrak{U}^{\prime}(\lambda)$. Especially when $n=2$, always $\mathfrak{U}^{\prime \prime}(\lambda)=\{0\}$ and $\mathfrak{U}(\lambda)=\mathfrak{U}^{\prime}(\lambda)=\mathfrak{C}(\lambda)$. When $n \geqslant 3, \mathfrak{U}^{\prime \prime}(\lambda)=\{0\}$ or $\neq\{0\}$ according as $\lambda$ is regular or not.

We proved in $\S 4$ and $\S 5$ that on $G=S L(n, R) \Subset\left(\lambda_{\mu}\right)=\mathfrak{A}^{\prime}\left(\lambda_{\mu}\right)$ for any imaginary $\mu \in\left(\mathfrak{h}_{c}^{0}\right)^{*}$.

\section{$\S 6$. The case of complex semi-simple Lie groups}

In this section let $G$ be a connected complex semisimple Lie group and $H=H^{0}$ its Cartan subgroup. Then we can apply Prop's 1 and 2. For any root $\alpha$ of $(\mathfrak{g}, \mathfrak{h})$, define $H_{\alpha} \in \mathfrak{h}$ as $\alpha(X)=\left\langle H_{\alpha}, X\right\rangle(X \in \mathfrak{h})$, where $\langle$,$\rangle denotes the Killing form of \mathfrak{g}$. Let $X \rightarrow \bar{X}(X \in \mathfrak{h})$ be the conjugation of $\mathfrak{h}$ with respect to the real subalgebra spanned by $H_{\alpha}(\alpha \in P)$. Denote by $\mathfrak{h}^{*}$ the dual space of $\mathfrak{h}$ over $C$. Then any character $\chi$ of $H$ can be expressed uniquely as

$$
\chi(\exp X)=\exp \{p(X)+q(\bar{X})\} \quad(X \in \mathfrak{h})
$$

where $p, q \in \mathfrak{h}^{*}$. (Note that $H$ is connected.) Denote $\chi$ by $(p, q)$ and consider it also as an element of $\mathfrak{h}^{*}$. Let $W$ be the Weyl group of $(\mathfrak{g}, \mathfrak{h})$. It operates on $\chi=(p, q)$ as $w \chi=(w p, w q)(w \in W)$. The Weyl group $W_{c}$ of $\left(\mathfrak{g}_{c}, \mathfrak{h}_{c}\right)$ is isomorphic to $W \times W$ in such a way that $\sigma=\left(w, w^{\prime}\right)\left(w, w^{\prime} \in W\right)$ operates on $\chi=(p, q)$ as $\sigma \chi=\left(w p, w^{\prime} q\right)$. Let $T^{\chi}$ be the induced representation of $\chi$ on a Hilbert space $\mathscr{H}^{\chi}$ defined in $\S 2$ and $\pi^{\chi}$ its character. Let $J\left(\mathfrak{h}_{c}\right)$ be the set of $W$-invariant analytic differential operators on $H$. Then $I\left(\mathfrak{h}_{c}\right)$ is generated by $J\left(\mathfrak{h}_{c}\right)$ and $\overline{J\left(\mathfrak{h}_{c}\right)}$. We see from these facts that $Z \pi^{\chi}=\lambda_{\chi}(Z) \pi^{\chi}(Z \in 3)$, and that $\pi^{\chi}=\pi^{\chi^{\prime}}$ (or $\lambda_{\chi}=\lambda_{\chi^{\prime}}$ ) if and only if $\chi^{\prime}=w \chi($ or $=\sigma \chi)$ for some $w \in W$ (or $\left.\sigma \in W_{c}\right)$. A study of the equations (1.4) and (1.5) gives us the following

Lemma 6.1. For any character $\chi=(p, q), \quad \mathfrak{U}\left(\lambda_{\chi}\right)=\mathfrak{U}^{\prime}\left(\lambda_{\chi}\right)+$ 
$\mathfrak{Y}^{\prime \prime}\left(\lambda_{\chi}\right)$ (direct sum) and $\mathfrak{X}^{\prime}\left(\lambda_{\chi}\right)$ is spnned by $\left\{\pi^{\chi^{\prime}} ; \chi^{\prime}=(p, w q), w \in W\right\}$, whence $\sqrt{ }\left(\lambda_{\chi}\right) \supset \mathfrak{X}^{\prime}\left(\lambda_{\chi}\right)$.

We want to prove $\mathfrak{S}\left(\lambda_{\chi}\right)=\mathfrak{A}^{\prime}\left(\lambda_{\chi}\right)$. Meanwhile we obtain from [3(b), App. II] $\left(^{*}\right)$ the following

Proposition 9. Let $G$ be any of $S L(n, \boldsymbol{C}), S O(2 n+1, \boldsymbol{C}), S p(n$, $C)$ and $S O(2 n, C)$. When $n=2$ or $3, \mathfrak{U}^{\prime \prime}(\lambda)=\{0\}$ for any $\lambda$. When $n \geqslant 4$, there always exist some $\lambda$ for which $\mathfrak{U}^{\prime \prime}(\lambda) \neq\{0\}$. Moreover $\mathfrak{H}^{\prime \prime}(\lambda)=\{0\}$ for any $\lambda=\lambda_{\chi}$ with imaginary $\chi=(p, q) \in \mathfrak{h}_{c}^{*}$.

As a corollary of the last assertion of this proposition, we obtain

Theorem 2. For any complex classical group $G$, a tempered invariant eigendistribution of $G$ is always a linear combination of the characters of its irreducible unitary representations.

Now, to determine $\sqrt{ }(\lambda)$, we apply Lem's 2.1 and 2.2 and some results of D. P. Zhelobenko in $[4(\mathrm{a}),(\mathrm{b})]$. Suppose, for simplicity, that $G$ is simply connected. Then a pair of $p, q \in \mathfrak{h}^{*}$ defines a character of $H$ if and only if $p_{\alpha}-q_{\alpha}$ is integer for any $\alpha \in P$, where $p_{\alpha}=2<p, \alpha>1$ $\langle a, a\rangle$. A character $\chi=(p, q)$ is called discretely positive if for any $\alpha \in P, p_{\alpha}$ and $q_{\alpha}$ are not negative integers at the same time. D. P. Zhelobenko [4(a), §11] defined for any discretely positive character $\chi$, "the minimal representation $\mu(\chi)$ " as the restriction of $T^{\chi}$ on an invariant subspace $\mathscr{N}^{\chi}$ of $\mathscr{H}^{\chi}$ with a stronger topology than the one induced from $\mathcal{H}^{\chi}$ and proved the following facts.

Lemma 6.2. The representation $\mu(\chi)$ is completely irreducible in the sence of $R$. Godement and the two $\mu(\chi)$ and $\mu\left(\chi^{\prime}\right)$ are equivalent if and only if there exists some $w \in W$ such that $\chi^{\prime}=w \chi[4(\mathrm{a}), \S 11]$. Any quasi-simple irreducible representation of $G$ is infinitesimally equivalent to some $\mu(\chi)$ [4(b), Th. 7]. 
Define the character of $\mu(\chi)$ as that of the restriction of $T^{\chi}$ on the closure of $\mathscr{T}^{\chi}$ in $\mathscr{H}^{\chi}$ and denote it by $\bar{\mu}(\chi)$. Then it follows from Lem. 6.2 that for any $\lambda$, the set of all irreducible characters with infinitesimal character $\lambda$ does consist of all different $\bar{\mu}(\chi)$ with discretely positive $\chi$ such that $\lambda_{\chi}=\lambda$. This gives us the dimension of $\mathfrak{S}(\lambda)$. On the other hand, by Lem. 6.1, the dimension of $\mathfrak{U}^{\prime}(\lambda)$ is equal to the number of different $\pi^{\chi}$ such that $\lambda_{\chi}=\lambda$. Thus we see that $\operatorname{dim} \mathfrak{E}(\lambda)=\operatorname{dim}$ $\mathfrak{U}^{\prime}(\lambda)$, whence $\mathfrak{C}(\lambda)=\mathfrak{U}^{\prime}(\lambda)$.

Theorem 3. Let $G$ be a connected complex semisimple Lie group. For any $\lambda, \mathfrak{A}(\lambda)=\mathfrak{U}^{\prime}(\lambda)+\mathfrak{U}^{\prime \prime}(\lambda)$ (direct sum) and $\mathbb{E}(\lambda)=\mathfrak{A}^{\prime}(\lambda)$.

This theorem and Prop. 9 give us the following

Theorem 4. For $S L(n, C), S O(2 n+1, C), S p(n, C)$ and $S O(2 n$, $C)$, if $n \geqslant 4$, there always exist invariant eigendistributions on it which can not be expressed as linear combinations of irreducible characters. No such distribution is tempered.

(*) Errata. In [3(b), App. II]; p. 60, the 2 nd line from below should be " $p(t ; \rho)=p\left(\tau^{\prime} t ; \tau\right.$ ' $\left.\rho \tau\right)\left(\rho \in W, \tau \in \mathfrak{T}(c), \tau^{\prime} \in \mathfrak{T}(d)\right)$ "; p. 63, the right hand side of $\left(17^{\prime}\right)$ should be multiplied by $\prod_{j=1}^{n}\left(e^{z_{j}}-e^{-z_{j}}\right)$; p. 66, the 4 th and 5 th lines from below should be "in another cases, $p(t)$ is symmetric with respect to the union of $t_{j}\left(j \in A_{k}^{+} \cap B_{l}^{+}\right)$ and $-t_{j}\left(j \in A_{\bar{k}}^{-} \cap B_{l}^{-}\right)$and with respect to the union of $t_{i}\left(i \in A_{k}^{+} \cap\right.$ $\left.B_{l}^{-}\right)$and $-t_{i}\left(i \in A_{\bar{k}}^{-} \cap B_{l}^{+}\right)^{\prime \prime}$.

KYOTO UNIVERSITY

\section{References}

[ 1 ] I. M. Gel'fand and M. I. Graev: Unitary representations of the real unimodular groups (principal non-degenerate series). Izv. AN SSSR, Ser. Math., 17, 189-248 (1953). 
[2] Harish-Chandra:

(a) Representations of semisimple Lie groups. I, II, III. Trans. Amer. Math. Soc., 75, 185-243 (1953), 76, 26-65, 234-253 (1954).

(b) The characters of semisimple Lie groups. Ibid., 83, 98-163 (1956).

(c) A formula for semisimple Lie groups. Amer. J. Math., 79, 733-760 (1957).

(d) Invariant eigendistributions on a semisimple Lie group. Trans. Amer. Math. Soc., 119, 457-508 (1965).

(e) Discrete series for semisimple Lie groups. II. Acta Math., 116, 1-111 (1966).

[3] T. Hirai:

(a) The characters of some induced representations of semisimple Lie groups. J. Math. Kyoto Univ., 8, 313-363 (1968).

(b) Invariant eigendistributions of Laplace operators on real simple Lie groups. I. Japan. J. Math., 39, 1-68 (1970).

(c) Characters of representations and invariant eigendistributions on real semisimple Lie groups (in Japanese). Sugaku, 23, 241-260 (1971).

[4] D. P. Zhelobenko:

(a) Operational culculus on a complex semisimple Lie group. Izv. AN SSSR, Ser. Math., 33, 931-973 (1969).

(b) Classification of extremely irreducible and normally irreducible representations of a complex semisimple Lie group. Ibid., 35, 573-599 (1971). 